\title{
La evaluación docente en educación superior: características y desafíos
}

\section{The assessment of faculty in higher education: characteristics and challenges}

\section{ISSN 2071-8748 \\ E-ISSN 2218-3345 \\ (c) (i) (5) (0) \\ BY NC SA}

\author{
Marisol Esperanza Cipagauta-Moyano \\ Corporación Universitaria Minuto de Dios - UNIMINUTO \\ mcipagauta@uniminuto.edu \\ ORCID: 0000-0002-1378-8824 \\ Recibido: 23 de agosto 2019 \\ Aprobado: 10 de septiembre 2019
}

URI: http://hdl.handle.net/11298/1140

DOI: https://doi.org/10.5377/entorno.v0i68.8460

\section{Resumen}

La evaluación docente es un tema que atañe a la comunidad académica en general, cuyos resultados se emplean para el mejoramiento de los procesos en la enseñanza, el aprendizaje y el diseño de contenidos, principalmente. Evaluar el desempeño docente significa realizar un trabajo riguroso con el fin de tomar las medidas necesarias para fortalecer el quehacer en el ambiente de aprendizaje y, por ende, en los indicadores de calidad relacionados con este aspecto para la docencia universitaria en la gestión de operaciones. Los criterios, así como los instrumentos y modelos para evaluar el desempeño docente, pueden variar, y esto depende de varios factores: la metodología de estudio, presencial o a distancia, pues las mediaciones cambian al usar la tecnología como catalizadora entre el que enseña y el que aprende; los intereses administrativos de la institución; o bien la cultura de calidad definida, entre otros. La presente revisión se centra en la discusión de la evaluación docente realizada por los estudiantes como una invitación a

\section{Abstract}

The assessment of faculty members is a topic that concerns to the whole scholar community; its results are used for the betterment of the teaching process, learning, and the design of content topics, mainly. Assessing faculty performance means to conduct a rigorous work with the purpose of taking the necessary steps to strengthen the tasks in a learning environment and, therefore, in the quality indicators related to this aspect for university teaching in the management of operations. The criteria, as well as the models and tools to assess faculty performance may vary; this depends on different factors: the methodology used for the studyonline or regular-since the mediation changes when technology is used as an agent of change between the instructor and the learner; the administrative interests of the institution; or the defined culture of quality, among others. This review focuses on the discussion of the faculty evaluation carried out by the student body as an invitation to review and strengthen this process in higher

1 Doctora en Educación por Nova University, USA; magíster en Administración de Instituciones Educativas del Instituto Tecnológico y de Estudios Superiores de Monterrey, México. Comunicadora social-periodista de la Corporación Universitaria Minuto de Dios-UNIMINUTO. En la actualidad se desempeña como directora del Centro de Excelencia Docente aeiou de UNIMINUTO y profesora asociada de la maestría en educación de la misma institución. 
revisar y potencializar este proceso en las instituciones de educación superior con el ánimo de implantar una cultura de evaluación. La revisión bibliográfica se hace durante el periodo comprendido entre 2016 y 2019, con especial énfasis en artículos científicos que hacen referencia a la evaluación docente desde perspectivas particulares y generales en las que se incluyen trabajos tanto de tipo cuantitativo como cualitativo.

\section{Palabras clave}

Efectividad del docente - Evaluación, Pedagogía, Educación superior, Administración de personal docente, Calidad de la educación, Educación superior Formación profesional.

\section{Objetivo}

En el presente artículo de revisión bibliográfica se investiga sobre el tema de la evaluación docente en educación superior. El objetivo principal es analizar desde diferentes posturas conceptuales los aspectos más relevantes relacionados con la evaluación docente, sus protagonistas, modelos e instrumentos que se emplean durante el proceso, así como comprender la importancia que tiene realizar la evaluación como parte de la cultura de calidad en cualquier institución de educación superior y sus acciones de mejoramiento continuo. El análisis incluye la mirada del proceso desde la propia institución, la forma como los estudiantes la conciben, la relación con la formación continua de los docentes y los desafíos que se imponen como parte fundamental de procesos de mejoramiento que se incorporan en las instituciones de educación superior en un mundo cada vez más dinámico y cambiante, en la forma como se enseña, como se aprende y como se evalúa, todo esto permeado por las tecnologías emergentes.

\section{Desarrollo}

La metodología empleada para este artículo es descriptiva a partir de una revisión sistemática de la literatura relacionada con el tema de la evaluación docente en el nivel superior de la educación. Desde la indagación de cada una de las fuentes consultadas y con base en la experiencia de investigar sobre esta temática se hacen aportes con education institutions with the aim to implement a culture of assessment. The bibliographic review was conducted between 2016-2019, emphasizing mainly on scientific articles that make reference to faculty evaluation from specific and general perspectives which include both qualitative and quantitative work.

\section{Keywords}

Faculty effectiveness - Assessment, Pedagogy, Higher Education, Faculty administration, Quality of education, Higher Education - Professional training.

el objetivo de presentar características y desafíos para el proceso de evaluar la docencia como una práctica de calidad de las instituciones. El diálogo entre los autores es recurrente y la reflexión sobre sus apuestas es permanente a lo largo de este artículo de revisión. Se concluye con un apartado de contribuciones al respecto de la temática como un pretexto para seguir investigando sobre esta y empoderando el abordaje de la evaluación docente como un proceso fundamental, cuyos resultados son útiles para el mejoramiento de la calidad educativa y el desarrollo de estrategias de formación docente.

La evaluación docente en las instituciones de educación superior forma parte de los procesos inherentes a la actividad académica y todos aquellos de calidad que se implementan. "Es decir, la evaluación del profesorado es útil para proporcionar información que favorezca el mejoramiento profesional del docente y la toma de decisiones a nivel administrativo" (Márquez y Madueño, 2016, p. 58). De allí la importancia que las universidades le den y las acciones que emprenda por fortalecerla.

Si bien las instituciones incluyen en estos procesos la autoevaluación deldesempeño del profesoryla coevaluación, la realizada por los estudiantes es fundamental por cuanto son ellos los directos beneficiarios de la labor que sus docentes realizan. "La evaluación docente se caracteriza por valorar en algunos casos desde la satisfacción de los estudiantes, 
el desempeño de los profesores, sus habilidades, el uso de recursos psicopedagógicos, estrategias de aprendizaje e investigación, hasta su apego a políticas institucionales" (Flores, Gatica, Sánchez y Martínez, 2017, p. 97).

De acuerdo con estos autores, la evaluación del docente integra varios aspectos a tener en cuenta en el desempeño de este desde diversas miradas, y por ende la percepción cambia. En este sentido, si solo se tiene en cuenta la opinión del estudiantado, "el profesorado lo equipara a una espada de Damocles que cualquier estudiante puede calificarlo mal, sin una justificación de peso" (Arbesú y García, 2017, p. 172). Visto de esta manera, se pierde el sentido de una evaluación transparente para el profesor, por tanto, resulta un primer reto para la institución diseñar estrategias que permitan una evaluación justa, en donde no haya cabida a subjetividades en el momento de aplicar instrumentos que permitan recoger la percepción del estudiantado en cuanto al desempeño de sus docentes, pero también en velar por la formación de estos para que cumplan a cabalidad su papel en el proceso de enseñanza y aprendizaje. "En suma, se debe evaluar la docencia asumiendo su complejidad, grado de idoneidad y por la pertinencia de las acciones realizadas" (Guzmán, 2016, p. 294).

No se trata de tener docentes que solo sepan de su área de conocimiento, sino de formarlos para enfrentar los desafíos de una educación dinámica y cambiante en cuanto a metodologías de enseñanza, formas de evaluar y trato con sus estudiantes, incluso hacerlos partícipes del diseño mismo de los instrumentos. "Los expertos y directivos coinciden en que los profesores debieran ser considerados en el diseño e implementación de los procesos de evaluación docente" (Arbesú y García, 2017, p. 173). Una vez esto ocurra, las instituciones contarían con una evaluación más democrática y participativa, que puede incluir aspectos no tenidos en cuenta hasta ahora de acuerdo con la forma tradicional de hacer el proceso. "La evaluación de la actividad docente, al igual que la realizada a las instituciones, programas o procesos, no está exenta de llevar a cabo su propio proceso de valoración y permanente perfeccionamiento de la labor docente en el contexto universitario" (Ramos, Ruiz, Pulido y Marín, 2019, p. 28).

En una investigación realizada por Arbesú y García (2017), los resultados mostraron que los funcionarios de las instituciones de educación superior no comprenden con claridad el significado de la evaluación docente; se han quedado simplemente en identificar distintos usos de esta entendida como sinónimo de cuestionarios que responden los estudiantes, una rutina de la burocracia, un tema relacionado con el salario, aspectos que son rechazados por los alumnos (p. 171). En este aspecto, es necesario revaluar si lo que se pretende es darle el lugar que le corresponde a la evaluación docente en la educación superior, que vaya más allá de la simple aplicación de instrumentos por cumplir con una normativa, se requiere del concurso de todos los actores para que el proceso sea válido y confiable. Una evaluación del desempeño docente dentro del ambiente de aprendizaje debe mostrar un panorama auténtico de lo que se hace o deja de hacer, todo esto con el propósito de mejorar la calidad educativa, por tanto, es indispensable brindar al profesor una retroalimentación de su labor, a partir de los resultados de la mencionada evaluación, para poder implementar las mejoras necesarias en ella (Martínez, Guevara y Valles, 2016, p. 132).

Hasta aquí, se ha reflexionado principalmente desde la posición de la universidad frente al proceso de la evaluación docente y su uso, pero es preciso hacer énfasis también en las estrategias de formación de los profesores para que fortalezcan su labor como docentes, y de esta forma conseguir mejores resultados al momento de aplicar instrumentos que recogen la percepción de los estudiantes con respecto al desempeño de sus docentes. Un plan de formación docente es necesario, por cuanto permite actualizarse en tendencias educativas, en la apropiación de tecnologías en el ambiente de aprendizaje, en el empoderamiento de su papel como mediador de la gestión del conocimiento que facilite "indagar en nuevas y eficaces formas de desarrollar en los alumnos el deseo y el interés por construir su propio conocimiento" (Fernández, Rodríguez y Fernández, 2016, p. 96).

La educación del siglo XXI plantea nuevos desafíos para los docentes no solo en la adquisición de nuevos conocimientos en su área de desempeño, sino porque su papel ha cambiado; pasó de ser el profesor que tenía todo el saber y lo transmitía a ser un guía del proceso de enseñanza y aprendizaje, lo cual significa un cambio también en la forma como se evalúa su oficio desde la perspectiva del estudiante. "La evaluación es una oportunidad para aclarar qué es lo que se quiere del profesor y definir cuáles son las condiciones necesarias que podrían hacer posible el cumplimiento de su labor" (Beltrán, 2018, p. 157). 
Bajo este panorama, entran en juego otros factores que antes no eran tenidos en cuenta porque simplemente no tenían injerencia mayor en el quehacer docente. "La calidad de una institución educativa universitaria depende fundamentalmente de la calidad humana, pedagógica y científica de sus docentes por eso ha sido una constante preocupación de las universidades la preparación y capacitación de sus profesores" (Castillo, 2017, p. 67). Solo por mencionar algunos, está el hecho de que el docente debe desenvolverse hoy en día en un mundo digitalizado en donde la educación no es ajena, antes bien, se vale de la tecnología para acercar a las personas y estas puedan estudiar incluso sin necesidad de desplazarse de un lugar a otro.

Así las cosas, la evaluación docente constituye un tema de prioridad en las agendas de las instituciones de educación superior, darle el lugar que le corresponde y hacer partícipes activos a todos los involucrados es una tarea prioritaria si se tiene en cuenta que la calidad con que este proceso se desarrolle redunda en beneficios para toda la comunidad educativa. Como dice Olivos (2018): "La calidad de la educación superior depende, entre otros factores, de la calidad de la enseñanza y, esta, a su vez, se ve fuertemente influida por la calidad del docente" (p. 88).

\section{Conclusiones}

La evaluación docente constituye, para las instituciones de educación superior, una oportunidad de identificar las fortalezas y el área de mejora del desempeño de sus profesores, esto significa que de la importancia que se le dé dependen procesos relacionados con indicadores de calidad en las instituciones, lo que significa un mejoramiento permanente en sus procesos. No es simplemente tabular los resultados de una evaluación y premiar al mejor docente evaluado por sus alumnos, sino de hacer un análisis de las opiniones y percepciones sobre el desempeño real de los docentes y cómo fortalecerlo o mejorarlo (Cipagauta, 2017, p. 21).

Una vez analizadas las falencias del desempeño docente, es tarea primordial de las instituciones revisar sus planes de acción y diseñar estrategias de formación, o las que convengan, todo con el ánimo de que sus profesores corrijan y mejoren su quehacer diario no solo en conocimientos de su área, sino en competencias relacionadas con el trabajo en equipo, la comunicación asertiva y el manejo de tecnologías, entre otras. Como dicen Loureiro, Míguez y Otegui (2016): "Sensatamente, la buena enseñanza se preocupa por la construcción de aprendizajes en los que se imbrican de modo interactivo tanto los procesos racionales como otros de carácter emocional" (p. 58).

Los resultados de la evaluación docente alertan sobre aspectos relacionados con los contenidos curriculares, pues varios de los criterios que se incluyen en los instrumentos de evaluación expresan puntos clave a tener en cuenta en este campo. Parte del éxito del desempeño de un docente está precisamente en el diseño de contenidos, si estos son claros la labor del docente se facilita y la comprensión por parte de los estudiantes también. En este sentido, Villaorroel y Bruna (2017) sostienen: “Deben preocuparse no solo de formar en contenidos a los futuros profesionales, sino que también invertir en esfuerzos por lograr perfiles de egreso de alta complejidad, que incluyen el desarrollo de procedimientos, habilidades de pensamiento de orden superior, y actitudes" (p. 92).

Lograr que la evaluación docente tenga una importancia significativa en la consolidación de una cultura de la calidad dentro de la institución de educación superior necesita del concurso de la toda la comunidad, es decir, de los administrativos, los directivos, los docentes y de los mismos estudiantes. El porcentaje de estos últimos, cuando llega el momento de evaluar a sus docentes, sigue siendo bajo. "La evaluación del profesorado como estrategia de mejora de la calidad de la enseñanza universitaria" (Tejedor, 2018, p. 3). No basta con evaluar la docencia, hacer un análisis de la evaluación del aprendizaje o la gestión del conocimiento, además es necesario comprobar el aporte del docente al cumplimiento de la misión de la universidad en un periodo determinado (Alija, Ramos y Pérez, 2017, p. 70).

Evaluar para mejorar ha de ser una constante en las instituciones de educación superior, por tanto, no se trata de un ejercicio que se haga solo al final de cada periodo académico, sino que sea también de corte formativo, la que, a lo largo del desarrollo de las actividades académicas, se efectúe a través de mecanismos como una sencilla encuesta de percepción para poder dar solución inmediata y efectiva a problemas que se estén presentando. "La evaluación sirve para estimar la calidad, entendiendo que el mero hecho de realizar controles no va a mejorarla" (Canos-Darós, Morera, Mascarell y Vidal-Carreras, 2017, p. 68). Tratándose de un proceso complejo por las variables que se involucran y 
de vital importancia para la formación de profesionales, la docencia debiera ser siempre tema de reflexión tanto individual como colectiva por parte de todos aquellos que se relacionan con esta (Contreras, 2018, p. 91).

Preguntas como qué, cómo, para qué, por qué y cuándo evaluar deben plantearse dentro de la cultura de calidad de una institución que le da la importancia necesaria al proceso de evaluar la docencia no como una obligación, sino como una estrategia de mejora continua en la que sus resultados evidencian cambios sustanciales en el desempeño de sus docentes. "Se entiende que un buen profesor universitario es garantía de excelencia educativa y una oportunidad para formar excelentes profesionales" (Castilla, 2018, p. 39).

\section{Referencias}

Acosta Ramos, A. C., Jiménez Ruíz, L. K., Pulido, E. G, y Redondo Marín, M. P. (enero-julio, 2019). Estrés ocupacional y evaluación de desempeño en docentes universitarios del departamento del Cesar, Colombia. Revista Encuentros, 17(1), 24-33. doi: http://dx.doi. org/10.15665/encuent.v17i01.1595

Alija, T., García Ramos, J. M., y Muñoz Pérez, S. (2016). Diseño de un modelo de evaluación y desarrollo docente en una universidad privada. Revista Complutense de Educación, 28(1), 61-80. Recuperado de https://doi. org/10.5209/rev_RCED.2017.v28. n1.48718

Arbesú García, M. I. y García Garduño, J. M. (2017). La evaluación docente en jaque, la visión de los funcionarios universitarios. Revista Iberoamericana de Evaluación Educativa, 10(2), 171-185. Recuperado de https://dialnet.unirioja.es/servlet/articulo ?codigo $=6205629$

Rueda Beltrán, M. (2018). Los retos de la evaluación docente en la universidad. Publicaciones. Facultad de Educacion y Humanidades del Campus de Melilla, 48(1), 171-192. doi: $10.30827 /$ publicaciones. v48i1.7334

Canós-Darós, L., Pons Morera, C., Santandreu Mascarell, C., y Vidal-Carreras, P. I. (2017). La evaluación de la enseñanza: algunas reflexiones. Working Papers on Operations Management, 8, 67-71. Doi: https://doi. org/10.4995/wpom.v8i0. 7163

Castilla Devoz, H. (2018). Más allá de los conocimientos: el arte de ser profesor mediador en el proceso de aprendizaje. Bogotá, Colombia: UNIMINUTO.

Cipagauta Moyano, M. E. (2017). Evaluar la docencia para mejorar la enseñanza. Bogotá, Colombia: UNIMINUTO
Contreras, G. (2018). Retroalimentación por pares en la docencia universitaria. Una alternativa de evaluación formativa. Formación Universitaria, 11(4), 83-94. doi: http://dx.doi.org/ 10.4067/S071850062018000400083

Fernández Díaz, M. J., Rodríguez Mantilla, J. M. y Fernández Cruz, F. J. (2016). Evaluación de competencias docentes del profesorado para la detección de necesidades formativas. Bordón Revista de Pedagogía, 68(2), 85101. Recuperado de https://dialnet.unirioja.es/servlet/ articulo?codigo $=5422161$

Flores Hernández, F., Gatica Lara, F., Sánchez Mendiola, M, y Martínez González, A. (2017). Evolución de la evaluación del desempeño docente en la Facultad de Medicina; evidencia de validez y confiabilidad. Investigación en educación médica, 6(22), 96-103. Doi: https://dx.doi.org/10.1016/j.riem.2016. 06.004

Guzmán, J. C. (2016). ¿Qué y cómo evaluar el desempeño docente? Una propuesta basada en los factores que favorecen el aprendizaje. Propósitos $y$ representaciones, 4(2), 285-358. Doi: http://dx.doi. org/10.20511/pyr2016.v4n2.124

Heras Castillo, V. E. (2017). La formación pedagógica del docente universitario. Palermo Business Review, (16), 65-73. Recuperado de https://www.palermo.edu/ economicas/cbrs/pdf/pbr16/PBR_16_04.pdf

Lemos, M., Calle, G., Roldán, T., Valencia, M., Orejuela, J., y Román Calderón, J. P. (2019). Factores psicosociales asociados al estrés en profesores universitarios colombianos. Diversitas: Perspectivas en Psicología, 15(1), 1-12. Doi: http://dx.doi.org/https://doi. org/10.15332/s1794-9998.2019.0015.05

Loureiro, S., Míguez, M., y Otegui, X. (2016). Desempeño docente en la enseñanza universitaria: análisis de las opiniones estudiantiles. Cuadernos de Investigación Educativa, 7(1), 55-67. Recuperado de http://www.scielo. edu.uy/scielo.php?script=sci__arttext\&pid=S168893042016000100005\&lng=es\&tlng=pt

Márquez Ibarra, L. y Madueño Serrano, M. L. (2016). Propiedades psicométricas de un instrumento para apoyar el proceso de evaluación del docente universitario. Revista electrónica de investigación educativa, 18(2), 53-61. Recuperado de http://www. scielo.org.mx/scielo.php?script=sci_arttext\&pid=S160740412016000200004\&lng=es\&tlng=es

Martínez Chairez, G. I., Guevara Araiza, A., y Valles Ornelas, M. M. (2016). El desempeño docente y la calidad educativa. Ra Ximhai, 12(6), 123-134. Recuperado de https://www.redalyc.org/articulo.oa?id=46148194007 
Moreno Olivos, T. (2018). La evaluación docente en la universidad: Visiones de los alumnos. Revista Iberoamericana Sobre Calidad, Eficacia y Cambio en Educacion, 16(3), 87-101. Doi: http://dx.doi. org/10.15366/reice2018.16.3.005

Tejedor Tejedor, F. (2018). La evaluación del profesorado como estrategia de mejora de la calidad de la enseñanza universitaria. Revista de Educacion $y$
Derecho, (17). Recuperado de https://dialnet.unirioja. es/servlet/articulo?codigo $=6375461$

Villarroel, V. y Bruna, D. (2017). Competencias pedagógicas que caractetizan a un docente universitario de excelencia: Un studio de caso que incorpora la perspectiva de docentes y estudiantes. Formación universitaria, 10(4), 75-96. Doi: https://dx.doi. org/10.4067/S0718-50062017000400008 